\title{
B-vitamin status in relation to cognitive health over 4 years in healthy older adults
}

\author{
C. F. Hughes ${ }^{1}$, M. Ward ${ }^{1}$, L. Hoey ${ }^{1}$, F. Tracy ${ }^{2}$, A. Molloy ${ }^{3}$ and H. McNulty ${ }^{1}$ \\ ${ }^{1}$ Northern Ireland Centre for Food and Health, University of Ulster, Coleraine, BT52 1SA, ${ }^{2}$ Causeway Hospital, \\ Coleraine, BT52 HIS and ${ }^{3}$ School of Biochemistry and Immunology, Trinity College Dublin, Dublin 2, Ireland.
}

Advancing age is associated with a decline in cognitive function which can range from mild cognitive impairment to dementia. Epidemiological evidence suggests that sub-optimal B-vitamin status may be associated with greater cognitive impairment in both healthy older adults and in patients with dementia ${ }^{(1)}$. In addition, evidence from randomised control trials has demonstrated a beneficial effect of B-vitamin supplementation on cognitive function ${ }^{(2,3)}$ but the evidence is not entirely consistent ${ }^{(4)}$. The aim was to investigate B-vitamin status in relation to cognitive decline over a 4 year follow-up period in healthy older adults. We hypothesised that poorer B-vitamin status at baseline would lead to a greater than expected rate of cognitive decline.

In a retrospective study, healthy older adults $(n=154$; aged 60-88years) who had been previously screened for cognitive function were reassessed four years after initial assessment. Cognitive function was assessed at both timepoints by the Mini-Mental State Examination (MMSE), the most widely used cognitive screening tools in a clinical setting. Participants were initially recruited as being cognitively healthy at baseline, i.e. having an MMSE score between 25-30.

At the 4 year follow-up assessment, when participants were aged $73 \cdot 4 \pm 7 \cdot 1$ years, mean cognitive MMSE scores had declined from $29 \cdot 1 \pm 1 \cdot 3$ to $27 \cdot 5 \pm 2 \cdot 3(P<0 \cdot 001)$. Although most participants showed a typical rate of cognitive decline expected for healthy older adults (i.e. a decrease of $0 \cdot 2-0 \cdot 6 \mathrm{MMSE}$ points per year) ${ }^{(5)}$, cognitive decline occurred at an accelerated rate in a sub-set of participants (i.e. greater than 1 MMSE point per year; $n=38$ ). Baseline predictors of accelerated cognitive decline were investigated (Table).

\begin{tabular}{|c|c|c|c|c|}
\hline & $\begin{array}{c}\text { Beta } \\
(\beta)\end{array}$ & $\begin{array}{l}\text { Odds } \\
\text { Ratio }\end{array}$ & $95 \% \mathrm{CI}$ & $P$ \\
\hline \multicolumn{5}{|l|}{ Baseline Factors } \\
\hline Age & $0 \cdot 12$ & $1 \cdot 12$ & $(1 \cdot 06-1 \cdot 18)$ & $<0 \cdot 001$ \\
\hline Gender & $-0 \cdot 21$ & $0 \cdot 81$ & $(0 \cdot 38-1 \cdot 71)$ & 0.580 \\
\hline BMI & $0 \cdot 01$ & $1 \cdot 00$ & $(0.92-1.09)$ & 0.976 \\
\hline Education & $-0 \cdot 49$ & $0 \cdot 61$ & $(0 \cdot 26-1 \cdot 42)$ & $0 \cdot 254$ \\
\hline Smoking & $1 \cdot 17$ & $3 \cdot 23$ & $(0 \cdot 62-16 \cdot 72)$ & $0 \cdot 162$ \\
\hline \multicolumn{5}{|l|}{ B-vitamin Biomarker Status } \\
\hline $\begin{array}{l}\text { Plasma Hcy }(\mu \mathrm{mol} / \mathrm{l} ; 12 \cdot 8-25 \cdot 4 \\
\text { vs } 6 \cdot 1-12 \cdot 5)\end{array}$ & $-0 \cdot 43$ & 0.65 & $(0 \cdot 27-1 \cdot 57)$ & 0.335 \\
\hline $\begin{array}{l}\text { Red cell folate (nmol/1; 191-719 } \\
\text { vs 726-2206) }\end{array}$ & 0.67 & 1.95 & $(0 \cdot 87-4 \cdot 34)$ & $0 \cdot 104$ \\
\hline $\begin{array}{l}\text { Serum B12 (pmol/1; 118-231 vs } \\
\text { 233-672) }\end{array}$ & 0.02 & $1 \cdot 02$ & $(0 \cdot 45-2 \cdot 32)$ & $0 \cdot 971$ \\
\hline $\begin{array}{l}\text { Plasma PLP (nmol/1; } 15 \cdot 4-43 \cdot 3 \\
\text { vs 43.9-198.4) }\end{array}$ & $1 \cdot 40$ & $4 \cdot 06$ & $(1 \cdot 80-9 \cdot 23)$ & 0.001 \\
\hline
\end{tabular}

For each B-vitamin biomarker, lowest tertile (or highest tertileHcy) was compared with other two tertiles. Values were considered significant if $P \leq$ 0.05.Abbreviations; CI: confidence interval; Hcy: homocysteine.

After adjustment for age, a low baseline concentration of vitamin B6, as measured using pyridoxal-5-phosphate (PLP; $<43 \cdot 3$ nmol/1) was associated with a 4-fold higher risk of having accelerated cognitive decline. Neither folate nor vitamin B12 concentrations were significantly associated with cognitive decline. In conclusion, lower vitamin B6 status at baseline was strongly associated with an accelerated rate of cognitive decline over the 4 year period. Vitamin B6 may be an important (often overlooked) protective factor in maintaining cognitive function in ageing.

1. Herrmann W, Obeid R. (2011) ClinChem Lab Med 49, 343-4.

2. de Jager CA, Oulhaj A, Jacoby R, et al. (2012) Int J Geriatr Psychiatry 27, 592-600.

3. Durga J, van Boxtel MPJ, Schouten EG, et al. (2007) Lancet 369, 208-16.

4. Ford AH, Flicker L, Alfonso H, et al. (2010) Neurology 75, 1540-7.

5. Park HL, O'Connell JE, Thomson RG. (2003) Int J Geriatr Psychiatry 18, 1121-34. 